\section{Avaliação nutricional: descrição da concordância entre avaliadores}

\section{Nutrition assessment: the agreement between observers}

\section{Resumo}

Este estudo objetivou avaliar a concordância interobservadores de medidas antropométricas e avaliação subjetiva do estado nutricional em adultos hospitalizados. Pacientes internados em um hospital geral universitário tiveram peso atual, peso usual, altura, circunferência do braço, prega cutânea tricipital, percentual de perda de peso, Índice de Massa Corporal, circunferência muscular do braço e Avaliação Nutricional Subjetiva Global obtidos por avaliadores independentes. A concordância foi avaliada pelo coeficiente kappa, teste $t$ pareado e coeficiente de correlação intraclasse (ICC). Foram avaliados 102 pacientes, com idade de $53 \pm 15$ anos. Foi obtida boa correlação entre observadores para todos os métodos antropométricos $(\mathrm{ICC} \geq 0,86)$ e diferenças médias de pequena magnitude. As variáveis categorizadas mostraram concordância ótima para o Índice de Massa Corporal (kappa=0,98; $\mathrm{IC}_{95 \%}$ : 0,95-1,0), fraca para a Avaliação Nutricional Subjetiva Global (kappa=0,46; $\mathrm{IC}_{95 \%}: 0,31$ $0,60)$ e moderada para os percentis 5 e 10 da circunferência do braço ( $k a p p a=0,78$; $\left.\mathrm{IC}_{95 \%}: 0,65-0,91\right)$, circunferência muscular do braço (kappa=0,73; $\left.\mathrm{IC}_{95 \%}: 0,54-0,92\right)$ e prega cutânea tricipital (kappa $=0,65 ; \mathrm{IC}_{95 \%}$ : 0,48-0,82). Para muitos pacientes houve discrepâncias clinicamente relevantes no percentual de perda de peso, circunferência do braço, prega cutânea tricipital e circunferência muscular do braço. Embora estatisticamente a reprodutibilidade dos métodos antropométricos tenha sido boa, deve-se ser prudente ao empregá-los no contexto hospitalar, visto as diferenças encontradas, em especial na antropometria do braço e na Avaliação Nutricional Subjetiva Global.

Palavras-chave: Confiabilidade. Reprodutibilidade. Antropometria. Avaliação nutricional. Estado nutricional.

Fonte de financiamento: Fundo de Incentivo à Pesquisa e Eventos (FIPE-HCPA). Número do processo: 05-353. Bolsa de mestrado CAPES, no 42001013074p2, concedida através do Programa de Pós-Graduação em Epidemiologia da FAMED/UFRGS.

Endereço para correspondência: Mariur Gomes Beghetto. Hospital de Clínicas de Porto Alegre, Rua Ramiro Barcelos 2350, sala 635, 90035-007, Porto Alegre, RS. E-mail: mbeghetto@hcpa.ufrgs.br 


\section{Abstract}

The aim of this study was to assess agreement between observers in performing anthropometric measures and a subjective assessment of nutritional status in hospitalized adults. In a general university hospital, independent observers obtained patients' current weight, usual weight, height, arm circumference, triceps skin fold thickness, percentage of weight loss, Body Mass Index, muscle arm circumference, and Subjective Global Assessment. Agreement was analyzed using the Kappa coefficient, paired t test, and intra-class correlation coefficient. The sample consisted of 102 patients, whose mean age was $53 \pm 15$ years. Good correlations (ICC $\geq 0.86$ ) and low mean differences between observers were obtained for all anthropometric measures. Agreement in classifying patients into categories of Body Mass Index was very strong (kappa $=0.98$; 95\%CI:0.95-1.0), weak for Subjective Global Assessment (kappa=0.46; 95\%CI:0.31-0.60) and moderate for the $5^{\text {th }}$ and $10^{\text {th }}$ percentiles of arm circumference (kappa $=0.78$; 95\%CI:0.65-0.91), muscle arm circumference (kappa $=0.73 ;$ 95\%CI:0.54-0.92) and triceps skinfold thickness (kappa $=0.65$; 95\%CI:0.480.82 ). Several patients had clinically relevant discrepancies in percentage of weight loss, arm circumference, triceps skin-fold thickness, and muscle arm circumference. Although anthropometric methods showed good statistical reproducibility, we should be prudent in applying these measures in the hospital setting, since discrepancies were observed, especially in arm anthropometry and Subjective Global Assessment.

Keywords: Reliability. Reproducibility. Anthropometry. Nutrition assessment. Nutritional status.

\section{Introdução}

O diagnóstico nutricional de adultos hospitalizados deve se apoiar em uma lógica que seja capaz de descartar ou confirmar desnutrição, onde o raciocínio probabilístico é o centro do processo decisório. Para tanto, diferentes métodos são empregados, visando aumentar a probabilidade de se acertar o diagnóstico de desnutrição nos indivíduos que de fato estão desnutridos e descartar a presença de desnutrição nos indivíduos não desnutridos. Todo o processo, portanto, visa melhorar a interpretação deste conjunto de informações, diminuindo a incerteza envolvida quando se estabelece um diagnóstico, reduzindo os riscos de se intervir desnecessariamente em indivíduos que não precisam receber tratamento, e de se deixar de intervir em indivíduos que deveriam receber tratamento ${ }^{1,2}$. Neste sentido, não somente um método deve ser capaz de apontar para um mesmo diagnóstico quando repetido pelo mesmo investigador, em diferentes oportunidades, mas de apontar para um mesmo diagnóstico quando empregado por diferentes investigadores ${ }^{3}$.

Métodos subjetivos e objetivos são empregados nas rotinas de avaliação nutricional de adultos hospitalizados, sem que, contudo, tenham passado por validação apropriada para esta finalidade. A Avaliação Nutricional Subjetiva Global (ANSG) é um método subjetivo, essencialmente clínico e em forma de questionário, sujeita, portanto, ao treinamento do profissional que o aplica. A partir da impressão do avaliador, os pacientes são classificados em uma das 3 categorias: (A) nutridos, (B) moderadamente desnutridos ou com suspeita de desnutrição ou (C) desnutridos severos. A ANSG foi desenvolvida e validada a partir de um grupo de pacientes cirúrgi$\cos ^{4,5}$, sendo atualmente empregada na avaliação nutricional de diferentes grupos de pacientes clínicos e cirúrgicos ${ }^{6-9}$. Métodos antropométricos (como o Índice de Massa Corporal, prega cutânea tricipital e circunferência muscular do braço), desen- 
volvidos para avaliar sujeitos saudáveis, são amplamente empregados, de forma isolada ou como integrantes de escores, para a avaliação do estado nutricional de adultos hospitalizados ${ }^{9-13}$, ainda que possam apresentar limitações quanto à concordância intra e interobservadores ${ }^{3,14,15}$. Duerksen et al. ${ }^{16}$, ao empregarem a antropometria para avaliar 87 idosos e Hasse et al. ${ }^{17}$, ao empregar a ANSG em indivíduos cirróticos candidatos a transplante, encontraram apenas discreta concordância entre os observadores (kappa $=0,48$ e $\leq 0,5$, respectivamente).

Dentre as etapas de validação de métodos de avaliação nutricional, há necessidade de se verificar a concordância entre os observadores. Sendo assim, o objetivo do presente estudo, que integra um projeto de pesquisa maior, foi avaliar a concordância interobservadores na aferição de medidas antropométricas e da Avaliação Nutricional Subjetiva Global, na avaliação do estado nutricional de adultos hospitalizados.

\section{Pacientes}

Foram consecutivamente incluídos 102 adultos, internados por motivos clínicos e/ ou cirúrgicos nas unidades de internação de um hospital geral universitário de alta complexidade da região sul do Brasil. Não foram incluídos pacientes internados no centro de terapia intensiva, unidade de transplante de medula óssea, em uso de aparelho gessado, submetidos à amputação de membro, e os que apresentaram impossibilidade de informar sobre seu estado clínico ou de se submeter aos métodos antropométricos de avaliação do estado nutricional. $\mathrm{O}$ estudo foi aprovado pelo Comitê de Ética em Pesquisa da Instituição.

\section{Métodos}

A identificação dos pacientes potencialmente elegíveis foi realizada por meio do sistema informatizado de gerenciamento de pacientes do hospital e, entre 29 de janeiro e 23 de março de 2006, durante as primeiras 72 horas da internação hospitalar, foi realizada avaliação nutricional por dois avaliadores independentes, incluindo:

- $\quad$ peso usual (PU)

- peso atual (PA)

- percentual de perda de peso em 6 meses (PPP)

- altura corporal

- índice de massa corporal (IMC)

- circunferência do braço (CB)

- prega cutânea tricipital (PCT)

- circunferência muscular do braço (CMB)

- Avaliação Nutricional Subjetiva Global (ANSG).

Cada paciente foi avaliado em duplicada, por um nutricionista (avaliação adotada como padrão de referência) e por um de 7 acadêmicos do curso de graduação em nutrição previamente treinado (avaliação em teste), sendo todos cegos para a avaliação oposta à sua. $\mathrm{O}$ mesmo protocolo de pesquisa e a mesma rotina de avaliação foi adotada por todos avaliadores. Primeiramente, o aluno perguntou ao paciente sobre o seu $\mathrm{PU}$ e aplicou o instrumento da $\mathrm{ANSG}^{18}$. A seguir, aferiu $\mathrm{PA}$, altura corporal, $\mathrm{CB}^{19}$ e $\mathrm{PCT}^{20}$. Posteriormente foi procedida pela nutricionista a mesma seqüência para obtenção dos dados antropométricos e ANSG. Para aferição do peso foram utilizadas balanças eletrônicas tipo plataforma, com capacidade para até $200 \mathrm{Kg}$ e escala de 100g. Na aferição da altura foram utilizados antropômetros fixos, disponíveis nas unidades de internação. Tanto as balanças quanto os antropômetros são anualmente aferidos e certificados pelo Instituto Nacional de Pesos e Medidas do Brasil. Para a verificação da CB foi utilizada fita métrica não extensível com escala em milímetros, marca Barlow® (W. Germany, Brasil). Para a verificação da PCT foi utilizado plicômetro Lange Skinfold Calipter (btechnology Incorporated Cambridge, Maryland, EUA). Todas as medidas antropométricas foram realizadas em 3 tomadas e foi utilizado o valor médio. 
Foram adotadas as seguintes equações para cálculo do PPP, IMC e CMB, respectivamente: $\mathrm{PPP}^{21}=[(\mathrm{PA}-\mathrm{PU}) / \mathrm{PU}] \times \mathrm{x} 100$; $\mathrm{IMC}^{22}=\mathrm{PA}(\mathrm{Kg}) /$ altura $^{2}(\mathrm{~m}) ; \mathrm{e} \mathrm{CMB}^{23}(\mathrm{~cm})$ $=\mathrm{CB}(\mathrm{cm})-[\mathrm{PCT}(\mathrm{mm}) \times 0,314]$.

A concordância entre os avaliadores foi testada mediante a obtenção do coeficiente kappa, para as variáveis categóricas, e do gráfico de Bland \& Altman ${ }^{24}$ e gráfico proposto por $\mathrm{Lin}^{25}$, para as variáveis contínuas. A fim de descrever as diferenças entre os observadores, foi realizado teste $t$ pareado (diferença entre as médias) e coeficiente de correlação intraclasse, para as variáveis contínuas. Também, foram avaliados os percentuais de concordância entre os avaliadores, estabelecendo o que chamamos de "diferença clinicamente relevante”, considerando-se como aceitável uma variabilidade inerente ao momento da avaliação e desempenho dos instrumentos utilizados. Para tanto, aceitou-se diferenças de $500 \mathrm{~g}$ para PU e PA, $0,5 \mathrm{~cm}$ para altura, $0,5 \mathrm{~cm}$ para $\mathrm{CB}, 2$ a $10 \mathrm{~mm}$ para PCT, $1 \%$ no PPP, $0,5 \mathrm{Kg} / \mathrm{m}^{2}$ no IMC e $0,5 \mathrm{~cm}$ na CMB. O limite aceitável de diferença entre observadores na aferição da PCT segue o protocolo adotado pelo NHANES III $^{26}$. A tolerância aumenta em $2 \mathrm{~mm}$ a cada $10 \mathrm{~mm}$ da espessura da prega, ou seja: quando a prega mede de 0 a $10 \mathrm{~mm}$, o limite de tolerância da diferença é $2 \mathrm{~mm}$; quando mede 10 a $20 \mathrm{~mm}$, diferenças de até $4 \mathrm{~mm}$ são aceitáveis; se 20 a $30 \mathrm{~mm}$, aceitase diferenças de até $6 \mathrm{~mm}$; se 30 a $40 \mathrm{~mm}$, aceita-se $8 \mathrm{~mm}$ de diferença; e se maior que $40 \mathrm{~mm}$, aceita-se até $10 \mathrm{~mm}$ de diferença.

As análises foram conduzidas no pacote estatístico SPSS 12.0 e valores de $\mathrm{p}<0,05$ (bicaudal) foram considerados estatisticamente significativos.

\section{Resultados}

Foram avaliados 102 pacientes com média de idade de $53 \pm 15$ (19 a 88) anos, sendo $59 \%$ do sexo feminino e $56 \%$ hospitalizados por motivos cirúrgicos. Para todos os métodos foi observada forte correlação para os valores obtidos entre os ob- servadores (ICC $\geq 0,86)$ e diferenças médias de mínima magnitude, do ponto de vista clínico, ainda que para altura, PCT e CMB esta diferença seja estatisticamente significativa (Tabela 1).

Quando avaliada a concordância entre o observador de referência e o segundo observador, considerando-se 4 categorias para o percentual de perda de peso (Tabela 2), foi identificada moderada concordância entre os observadores (kappa $=0,74 ; \mathrm{IC}_{95 \%}$ : 0,63-0,85; $\mathrm{p}<0,001$ ), e plena concordância nas duas categorias que agruparam pacientes que perderam $>5 \%$ do peso corporal em 6 meses. Considerando-se a classificação do IMC também em 4 categorias (Tabela 2), foi identificada excelente concordância entre os observadores (kappa $=0,98$; $\mathrm{IC}_{95 \%}: 0,95$ 1,$0 ; \mathrm{p}<0,001$ ), quando somente um paciente foi classificado de modo discordante (classificado como eutrófico pelo avaliador de referência e como sobrepeso pelo avaliador 2). Em relação à ANSG, avaliada em 3 categorias (Tabela 2), foi obtida discreta concordância entre os observadores (kappa $\left.=0,46 ; \mathrm{IC}_{95 \%}: 0,31-0,60 ; \mathrm{p}<0,001\right)$. Ainda que nenhum paciente classificado como desnutrido grave pela avaliadora de referência tenha sido classificado como nutrido pela $2^{\text {a }}$ avaliadora, 16 pacientes classificados como nutridos pela avaliadora de referência foram erroneamente classificados com algum grau de desnutrição pela $2^{\mathrm{a}}$ avaliadora (Tabela 2).

Considerando-se os percentis usualmente adotados para classificação nutricional a partir da antropometria do braço, verificou-se moderada concordância entre os observadores para CB (kappa $\left.=0,78 ; \mathrm{IC}_{95 \%}: 0,65-0,91 ; \mathrm{p}<0,001\right)$, PCT (kappa $\left.=0,65 ; \mathrm{IC}_{95 \%}: 0,48-0,82 ; \mathrm{p}<0,001\right) \mathrm{e}$ CMB (kappa $=0,73 ;$ IC $_{95 \%}: 0,54-0,92$; $\mathrm{p}<0,001)$. Na Figura 1 está apresentada a proporção de concordância entre os avaliadores ao classificar o estado nutricional dos pacientes, de acordo com os percentis definidos por Frisancho et al. ${ }^{27}$, para CB, PCT e CMB. Para os 3 métodos, verificouse que o segundo avaliador tendeu a superestimar os valores obtidos pelo avalia- 
Tabela 1 - Valores médios, desvios-padrão, correlação e diferença entre as avaliações do observador de referência e do segundo observador, nas diferentes medidas antropométricas.

Table 1 - Mean values, standard deviations, correlation coefficients, and differences between observers for anthropometric measures.

\begin{tabular}{|c|c|c|c|c|c|c|c|}
\hline & $\mathrm{n}$ & $\begin{array}{l}\text { Observador } \\
\text { Referência }\end{array}$ & Observador 2 & $\mathrm{CCl}$ & $\begin{array}{l}\operatorname{Lin} \\
\left(\mathrm{IC}_{95 \%}\right)\end{array}$ & $\begin{array}{l}\text { Diferença } \\
\left(\mathrm{IC}_{95 \%}\right)\end{array}$ & $\mathrm{p} \#$ \\
\hline $\mathrm{PA}(\mathrm{Kg})$ & 96 & $68,5 \pm 16,5$ & $68,6 \pm 16,4$ & 0,999 & $\begin{array}{c}0,999 \\
(0,998-0,998)\end{array}$ & $\begin{array}{c}0,13 \\
(-, 03-0,29)\end{array}$ & 0,10 \\
\hline PU (Kg) & 100 & $70,6 \pm 16,0$ & $70,6 \pm 14,8$ & 0,971 & $\begin{array}{c}0,970 \\
(0,961-0,959)\end{array}$ & $\begin{array}{c}0,07 \\
(-, 67-0,82)\end{array}$ & 0,84 \\
\hline Altura $(\mathrm{cm})$ & 85 & $162,1 \pm 9,4$ & $162,1 \pm 9,4$ & 0,999 & $\begin{array}{c}0,999 \\
(0,999-0,999)\end{array}$ & $\begin{array}{c}-0,001 \\
(-0,002-0,000)\end{array}$ & $<0,01$ \\
\hline CB (cm) & 98 & $29,6 \pm 5,1$ & $29,5 \pm 4,9$ & 0,980 & $\begin{array}{c}0,979 \\
(0,973-0,972)\end{array}$ & $\begin{array}{c}-0,11 \\
(-0,31-0,09)\end{array}$ & 0,28 \\
\hline PCT (mm) & 98 & $16,3 \pm 9,0$ & $17,8 \pm 9,4$ & 0,893 & $\begin{array}{c}0,891 \\
(0,858-0,851)\end{array}$ & $\begin{array}{c}1,45 \\
(0,64-2,27)\end{array}$ & $<0,01$ \\
\hline PPP (\%) & 96 & $-3,5 \pm 9,36$ & $-3,8 \pm 10,1$ & 0,874 & $\begin{array}{c}0,840 \\
(0,791-0,782)\end{array}$ & $\begin{array}{c}-0,29 \\
(-1,29-0,69)\end{array}$ & 0,55 \\
\hline IMC $\left(\mathrm{Kg} / \mathrm{m}^{2}\right)$ & 82 & $26,3 \pm 5,4$ & $26,3 \pm 5,4$ & 0,998 & $\begin{array}{c}0,998 \\
(0,997-0,997)\end{array}$ & $\begin{array}{c}0,06 \\
(-0,01-0,13)\end{array}$ & 0,11 \\
\hline CMB (cm) & 98 & $24,5 \pm 3,5$ & $23,9 \pm 3,3$ & 0,865 & $\begin{array}{c}0,864 \\
(0,822-0,814)\end{array}$ & $\begin{array}{c}-0,57 \\
(-0,91--0,23)\end{array}$ & $<0,01$ \\
\hline
\end{tabular}

PA: Peso atual; PU: Peso usual; CB: Circunferência do braço; PCT: Prega cutânea tricipital; PPP: Percentual de perda involuntária de peso; IMC: Índice de Massa Corporal; CMB: Circunferência muscular do braço. CCI: Coeficiente de correlação intraclasse; Lin - Coeficiente de correlação de concordância proposto por Lin $25 ; \#$ : teste $t$ pareado.

PA: Actual weight; PU: Usual weight; CB: Arm circumference; PCT:Triceps skin-fold thickness; PPP: Percentage of weight loss; IMC: Body Mass Index; CMB: Muscle arm circumference; CCl: Intra-class correlation coefficient; Lin - Lin's concordance correlation coefficient ${ }^{25}$; \#: paired t test.

Tabela 2 - Concordância entre o observador de referência e observador 2 na classificação do percentual de perda de peso (kappa =0,74; IC $\mathrm{C}_{95 \%}: 0,63-0,85 ; \mathrm{p}<0,001$ ), Índice de Massa Corporal (kappa =0,98; IC ${ }_{95 \%}: 0,95-1,0 ; \mathrm{p}<0,001$ ) e Avaliação Nutricional Subjetiva Global (kappa $\left.=0,46 ; \mathrm{IC}_{95 \%}: 0,31-0,60 ; \mathrm{p}<0,001\right)$.

Table 2 - Agreement between observers in classifying weight loss (kappa $=0.74 ; 95 \% \mathrm{Cl}: 0.63-0.85 ; p<0,001$ ), Body Mass Index (kappa $=0.98 ; 95 \% C l: 0.95-1.0 ; p<0.001)$ and Subjective Global Assessment (kappa $=0.46 ; 95 \% C l: 0.31-0.60 ; p<0.001)$.

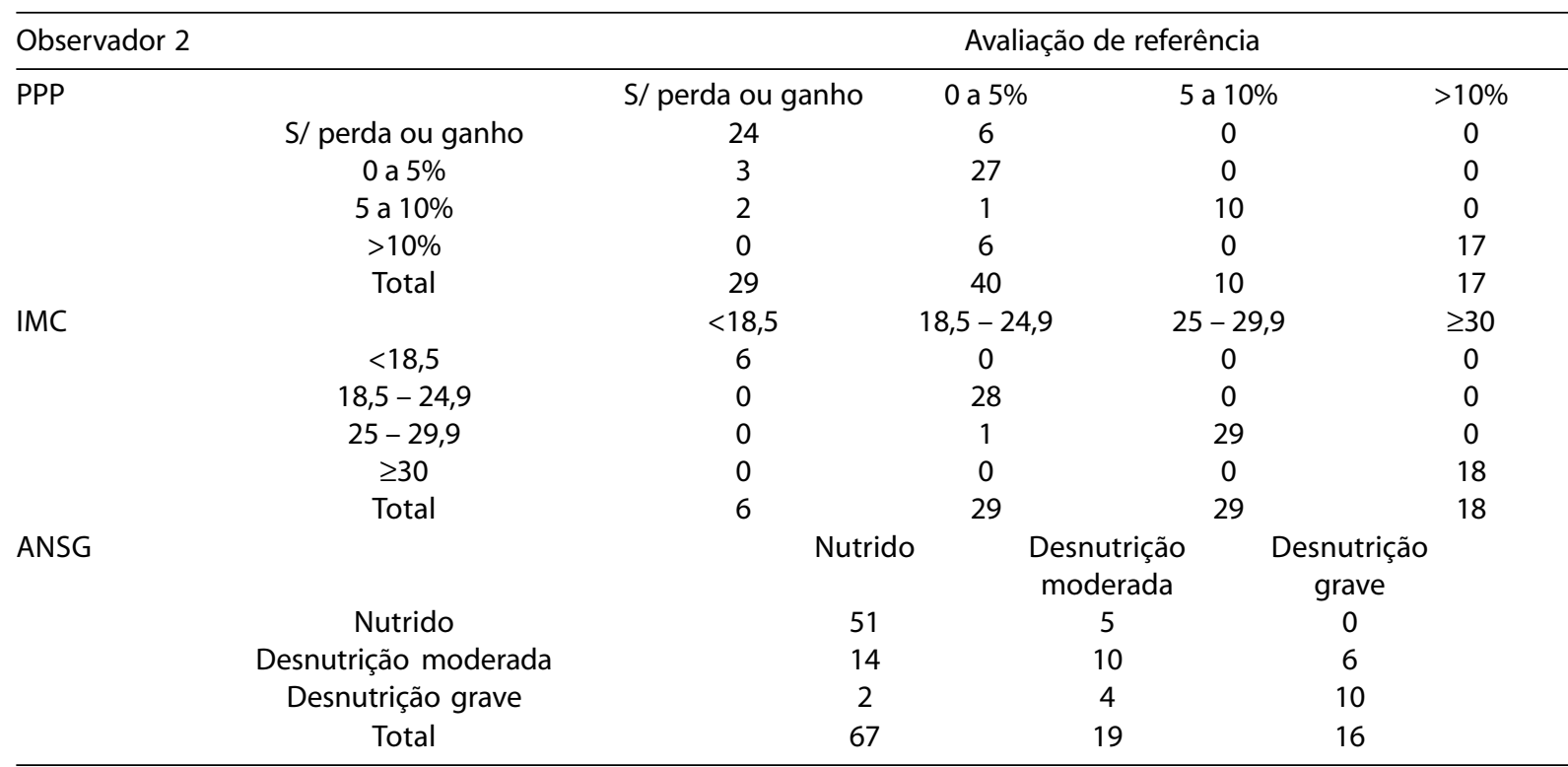

PPP: Percentual de perda de peso (\%); IMC: Índice de Massa Corporal (kg/m²); ANSG: Avaliação Nutricional Subjetiva Global. PPP: Percentage of weight loss (\%); IMC: Body Mass Index $\left(\mathrm{kg} / \mathrm{m}^{2}\right)$; ANSG: Subjective Global Assessment. 
dor de referência, classificando os pacientes em categorias de percentis acima da obtida pelo avaliador de referência. Notase que na categoria do percentil $\geq 10$, houve concordância para a quase totalidade dos pacientes, também para os 3 métodos.

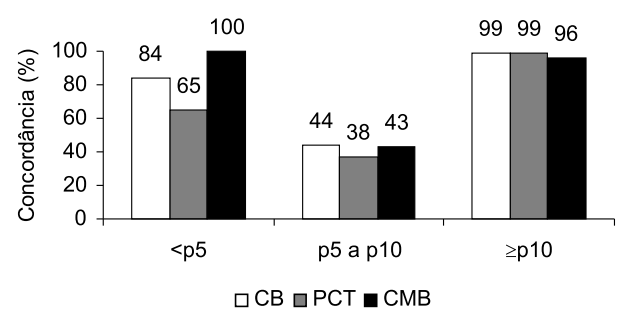

Figura 1 - Proporção de pacientes em que houve concordância (\%) entre os avaliadores na classificação dos percentis (p5 e p10), definidos por Frisancho et al. ${ }^{22}$, para circunferência do braço (CB), prega cutânea tricipital $(P C T)$ e circunferência muscular do braço (CMB).

Figure 1 - Proportion of agreement (\%) between observers in classifying patients according to the $5^{\text {th }}$ and $10^{\text {th }}$ percentiles ( $p 5$ and p10), defined by Frisancho et al. ${ }^{22}$, for arm circumference $(C B)$, triceps skin fold thickness $(P C T)$ and muscle arm circumference (CMB).

Os gráficos de Bland \& Altman (Figura 2) e Lin (Figura 3) sinalizam para grande concordância entre os observadores, especialmente para peso, altura, IMC e perda de peso. No entanto, ao se estabelecer critérios estritos, definidos como diferenças entre os observadores clinicamente não relevantes (até $500 \mathrm{~g}$, para PA e PU, até $0,5 \mathrm{~cm}$ para altura, CB e CMB, 2 a $10 \mathrm{~mm}$ para PCT, $1 \%$ para PPP e $0,5 \mathrm{Kg} / \mathrm{m}^{2}$ para IMC), o peso atual e a altura corporal (Figura 2a, 2c e 2g) mostraram boa concordância para o maior número de pacientes avaliados, enquanto que para $\mathrm{CB}$, PCT e CMB (Figura 2d, 2e e 2h) foram identificados valores fora destes parâmetros em maior percentual de pacientes.

\section{Discussão}

No presente estudo, encontrou-se alta concordância entre observadores treinados ao empregarem métodos antropo- métricos de avaliação do estado nutricional (PA, PU, altura, CB, PCT, CMB, PPP e IMC) em adultos hospitalizados não selecionados, enquanto a concordância na ANSG mostrou-se moderada. Porém, diferenças estabelecidas por nós como "clinicamente relevantes" foram identificadas em um grande percentual de pacientes na antropometria do braço, PPP e ANSG.

Assim como no presente estudo, elevada concordância entre observadores no emprego da antropometria e da ANSG foram anteriormente descritas ${ }^{28}$. Entretanto, são necessárias algumas considerações quanto à aplicação dos resultados estatísticos obtidos, sob o ponto de vista de seu emprego clínico. O coeficiente de correlação expressa, unicamente, a associação entre duas variáveis, não sendo, portanto, uma estatística apropriada para avaliar precisão e acurácia entre medidas. A diferença média e sua variabilidade expressam melhor o desempenho de cada método em relação è medida de referência, sendo, entretanto, afetadas por valores outliers. Assim, torna-se necessário avaliar a concordância entre os aferidores, para cada sujeito, sumarizando-se a concordância de modo dicotômico (concordância/discordância), mesmo quando certa margem de variabilidade é tolerada ${ }^{29}$. Isto parece claro quando analisamos a Figura 2 do presente estudo: quando adotados critérios estritos para a variabilidade esperada ("clinicamente relevantes") nota-se maior proporção de avaliações discordantes, enquanto ao se aceitar a variabilidade da amostra avaliada (de dois desvios padrão) a proporção de discordância é mínima.

Peso atual e altura foram as medidas onde em menor número de pacientes foram identificadas diferenças "clinicamente relevantes" entre os avaliadores. De fato, estas são as medidas antropométricas mais confiáveis em termos de reprodutibilidade $^{30}$ quando se assegura boa calibração e precisão dos instrumentos (balanças e antropômetros) e treinamento da equipe ${ }^{28}$, como implementado na nossa metodologia. Já em relação ao peso usual, gran- 
a)

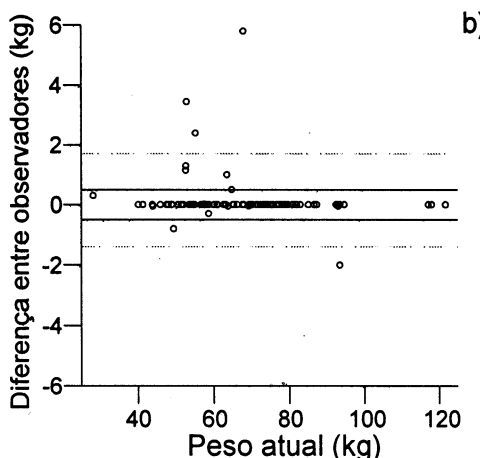

c)

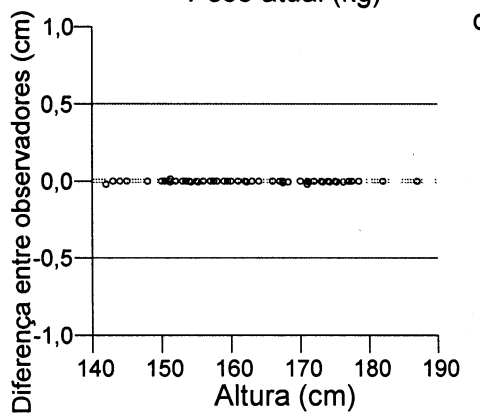

e)

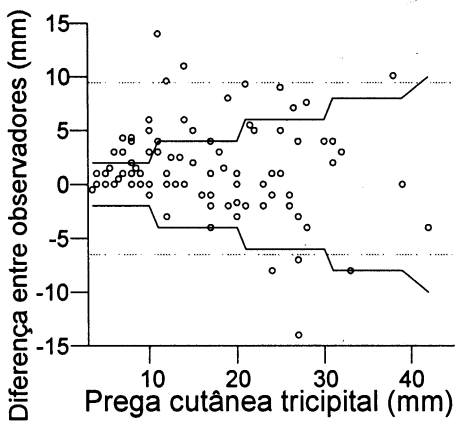

g)

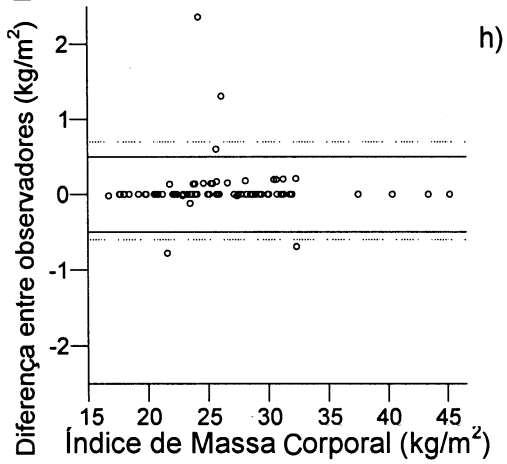

b)

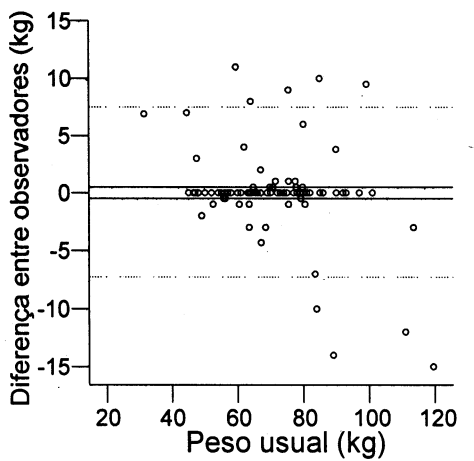

d)

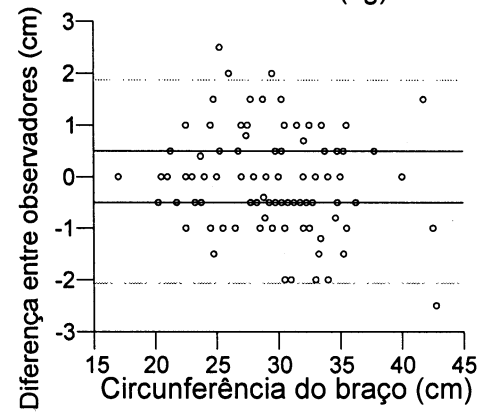

f)

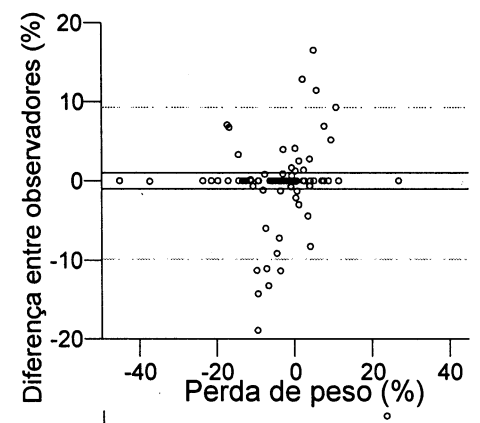

h)

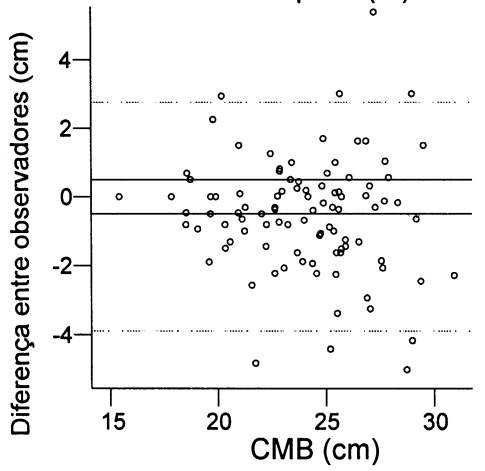

CMB - Circunferência muscular do braço.

Figura 2 - Diferença entre observadores nas medidas antropométricas (eixo Y), ao longo da média dos valores obtidos pelos dois observadores (eixo X). As linhas pretas apontam a tolerância de 0,5kg para peso atual (a) e peso usual (b), $0,5 \mathrm{~cm}$ para altura (c), circunferência do braço (d) e circunferência muscular do braço (e), 2 a $10 \mathrm{~mm}$ para prega cutânea tricipital (f), $1 \%$ para perda de peso (g) e 0,5Kg/m² para o Índice de Massa Corporal (h). As linhas cinzas indicam 1,96 desvios-padrão da média da diferença.

Figure 2 - Difference between observers in anthropometric measures ( $Y$-axis), in relation to the mean values obtained by the two observers (X-axis). Black lines point the tolerance of $0.5 \mathrm{~kg}$ for actual weight (a) and usual weight (b), $0.5 \mathrm{~cm} \mathrm{for} \mathrm{height} \mathrm{(c),}$ arm circumference $(d)$ and muscle arm circumference (e), 2 to $10 \mathrm{~mm}$ for skin-fold thickness (f), $1 \%$ for weight loss $(g)$ and $0.5 \mathrm{Kg} / \mathrm{m}^{2}$ for Body Mass Index (h). Gray lines represent 1.96 standard deviations to the mean difference. 
a)

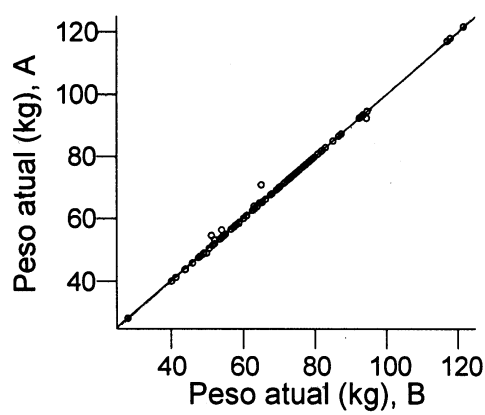

c)

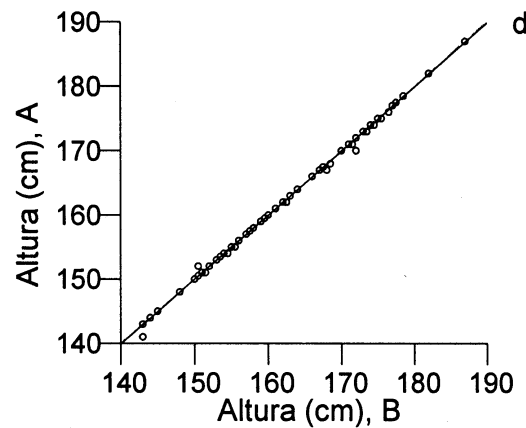

e)

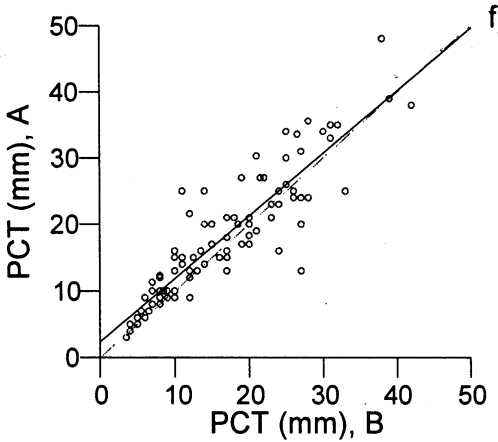

g)

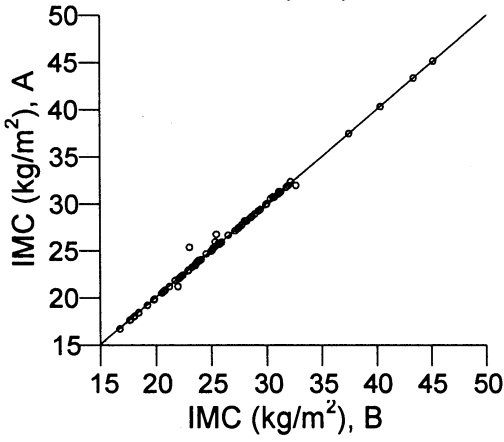

b)

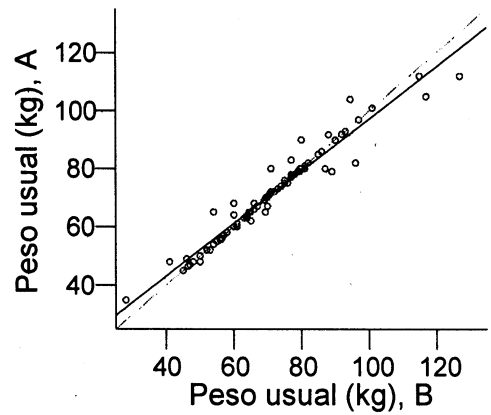

d)
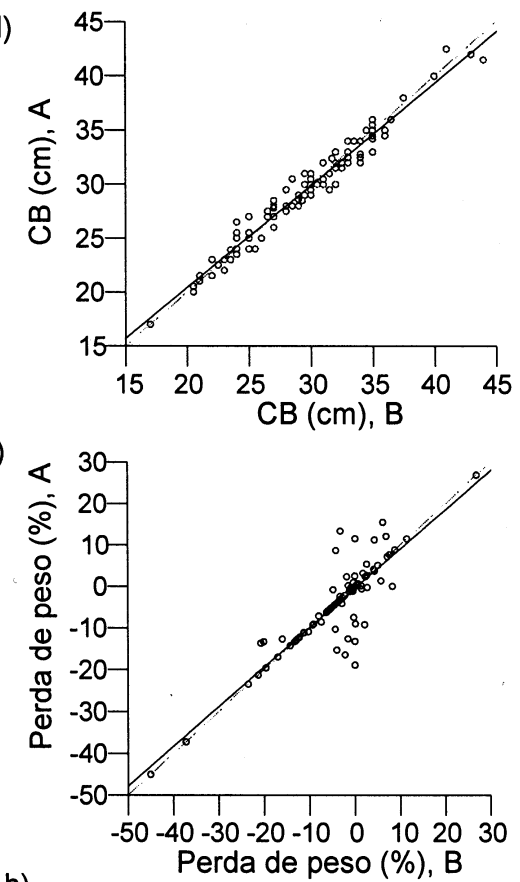

h)

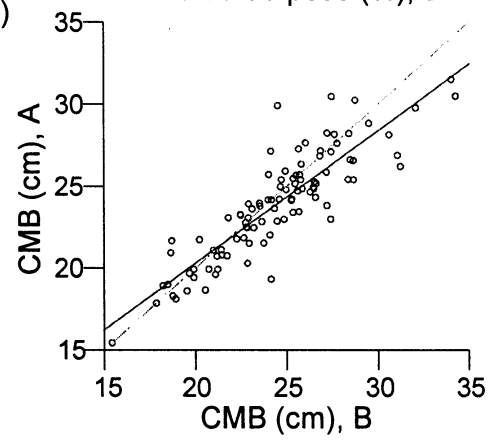

$A$ - Avaliação do aluno; B - Avaliação do nutricionista; $C B$ - Circunferência do braço; CMB - Circunferência muscular do braço; PCT - Prega cutânea tricipital; IMC - Indice de Massa Corporal.

Figura 3 - Concordância entre observadores ${ }^{25}$. As linhas cinzas sinalizam hipotética concordância perfeita (em ângulo de $45^{\circ}$ entre os eixos), enquanto as linhas pretas indicam a reta estimada para a concordância encontrada neste estudo. Cada ponto representa um paciente avaliado pelo aluno (A) e o observador de referência (B).

Figure 3 - Agreement between observers ${ }^{25}$. Gray lines point a hypothetical perfect agreement $\left(45^{\circ}\right.$ between axes), while black lines indicate the estimated line for the agreement found in this study. Each point represents a patient assessed by the student $(A)$ and the reference observer $(B)$. 
des diferenças ( $\geq 5 \mathrm{~kg}$ ) foram encontradas, o que poderia ser explicado por essa medida depender substancialmente da memória do paciente, como anteriormente demonstrado em grupos de indivíduos saudáveis e predominantemente jovens do Reino Unido ${ }^{31}$ e do Japão ${ }^{32}$, onde discrepâncias de até $10 \mathrm{~kg}$ foram encontradas.

A concordância na ANSG foi testada em um grupo selecionado de adultos submetidos à cirurgia eletiva do trato gastrintestinal, quando os autores encontraram moderada concordância entre os observadores (kappa: 0,78, IC95\%: 0,62-0,94; p<0,001) e recomendam que se teste a concordância interobservadores em cada meio ${ }^{18}$. Note-se que a ANSG sofre influência do viés recordatório relacionado ao peso usual e a todos os demais itens integrantes da ANSG, assim como pela experiência dos avaliadores, o que pode explicar a menor concordância entre observadores, no nosso estudo e no estudo de Duerksen em um hospital universitário do Canadá ${ }^{33}$. Por outro lado, maior concordância entre os observadores será identificada caso as categorias B e C da ANSG sejam agrupadas.

Burden et al. $^{34}$, ao avaliar a antropometria do braço, encontraram maior concordância entre os observadores, quando envolveram somente 2 avaliadores e pacientes provenientes de 4 unidades de internação em um hospital do Reino Unido. Ainda que no presente estudo o amplo conhecimento do protocolo de aferição das medidas por parte de todos avaliadores, como já descrito por Vegelin et al..$^{35}$, possa ter contribuído para melhorar a confiabilidade das medidas antropométricas, a acurácia da antropometria do braço depende, também, de características clínicas dos sujeitos avaliados: presença de edema, paresia e/ou plegia, estado de hidratação, restrição ao leito, entre outros, reforçando a necessidade de validação da concordância entre observadores, em diferentes contextos ${ }^{36}$.

A precisão de índices nutricionais é diretamente afetada pelas medidas antropométricas das quais derivam, o que explica a grande proporção de pacientes com valores e categorias de IMC concordantes. Por outro lado, em mais pacientes foram observados valores discordantes para o percentual de perda de peso e a circunferência muscular do braço, quando considerados valores "clinicamente relevantes", visto derivarem de medidas de maior divergência (peso usual e circunferência total do braço e prega cutânea, respectivamente) $)^{30,37}$. Mueller et al. ${ }^{38}$ já haviam descrito que índices compostos por diferentes medidas antropométricas são menos reprodutíveis que suas medidas originárias, salientando que isto deve ser levado em consideração, tanto na aplicação clínica quanto em investigações epidemiológicas.

De modo geral, há boa reprodutibilidade na classificação nutricional de adultos hospitalizados, quando avaliadores treinados empregam métodos antropométricos, perda de peso e ANSG. Ainda assim, para parte dos pacientes, pode haver divergência no diagnóstico nutricional, sugerindo a necessidade de que se valide o desempenho do avaliador e interavaliadores no emprego de cada método, em cada contexto hospitalar.

\section{Referências}

1. Belle GV, Fisher LD, Heagerty PJ, Lumley T. Biostatistics: A Methodology for the Health Sciences. $2^{\text {nd }}$ ed. New Jersey: Hoboken; 2004.

2. Sox HC. Common diagnostic tests, use and interpretation. Philadelphia: American College of Physicians; 1990.

3. Jones JM. Reliability of nutritional screening and assessment tools. Nutrition 2004; 20(3): 307-11.
4. Baker JP, Detsky AS, Wesson DE, Wolman SL, Stewart S, Whitewell J et al. Nutritional assessment: a comparison of clinical judgement and objective measurements. NEngl $J$ Med 1982; 306(16): 969-72.

5. Detsky AS, Baker JP, Mendelson RA, Wolman SL, Wesson $\mathrm{DE}$, Jeejeebhoy KN. Evaluating the accuracy of nutritional assessment techniques applied to hospitalized patients: methodology and comparisons. JPENJ Parenter Enteral Nutr 1984; 8(2): 153-9. 
6. Chung SH, Lindholm B, Lee HB. Is malnutrition an independent predictor of mortality in peritoneal dialysis patients? Nephrol Dial Transplant 2003; 18(10): 2134-40.

7. Correia MI, Campos AC. Prevalence of hospital malnutrition in Latin America: the multicenter ELAN study. Nutrition 2003; 19(10): 823-5.

8. Mello ED, Teixeira LB, Beghetto MG, Luft VC. Desnutrição hospitalar cinco anos após o IBRANUTRI Rev Bras Nutr Clin 2003; 18(2): 65-9.

9. Pirlich M, Schutz T, Norman K, Gastell S, Lubke HJ, Bischoff SC et al. The German hospital malnutrition study. Clin Nutr 2006; 25(4): 563-72.

10. Chen LK, Lin MH, Hwang SJ, Wang P, Chwang LC. Nutritional status and clinical outcomes among institutionalized elderly Chinese in Taiwan. Arch Gerontol Geriatr 2006; completar a referência.

11. de Luis D, Lopez Guzman A. Nutritional status of adult patients admitted to internal medicine departments in public hospitals in Castilla y Leon, Spain - A multi-center study. Eur J Intern Med 2006; 17 (8): 556-60.

12. de Ulibarri JI, Gonzalez-Madrono A, de Villar NG, Gonzalez P, Gonzalez B, Mancha A et al. CONUT: a tool for controlling nutritional status. First validation in a hospital population. Nutr Hosp 2005; 20(1): 38-45.

13. Rai A, Tewari M, Mohapatra SC, Shukla HS. Correlation of nutritional parameters of gallbladder cancer patients. $J$ Surg Oncol 2006; 93(8): 705-08.

14. Garcia SS, Pena CG, Lopez MXD, Cedillo TJ, Nunez ARC, Bearman SR. Anthropometric measures and nutritional status in a healthy elderly population. BMC Public Health 2007; 7:2 (no prelo). Disponível em http:// www.biomedcentral.com/1471-2458/7/2. Acessado em 27 de agosto de 2007.

15. Ulander K, Grahn G, Jeppsson B. Subjective assessment of nutritional status-validity and reliability of a modified Detsky index in a Swedish setting. Clin Nutr 1993; 12(1): 15-19.

16. Duerksen DR, Yeo TA, Siemens JL, O'Connor MP. The validity and reproducibility of clinical assessment of nutritional status in the elderly. Nutrition 2000; 16(9): $740-4$.

17. Hasse J, Strong S, Gorman MA, Liepa G. Subjective global assessment: alternative nutrition-assessment technique for liver-transplant candidates. Nutrition 1993; 9(4): 33943.

18. Detsky AS, McLaughlin J, Baker JP, Johnston N, Whittaker $\mathrm{S}$, Mendelson RA et al. What is subjective global assessment of nutritional status? JPEN J Parenter Enteral Nutr 1987; 11(1): 8-13.

19. Bishop CW, Bowen PE, Ritchey SJ. Norms for nutritional assessment of American adults by upper arm anthropometry. Am J Clin Nutr 1981; 34(11): 2530-9.
20. Frisancho AR. Triceps skin fold and upper arm muscle size norms for assessment of nutritional status. Am J Clin Nutr 1974; 27(10): 1052-8.

21. Dionigi R, Dominioni L. Predictive indices for the identification of high-risk patients. Eur Surg 1986; 18: 201-06.

22. World Health Organization. Physical Status: the use and interpretation of anthropometry. Tech Rep Ser 1995; 854: $1-452$.

23. Blackburn GL, Bistrian BR, Maini BS, Schlamm HT, Smith MF. Nutritional and metabolic assessment of the hospitalized patient. JPEN J Parenter Enteral Nutr 1977; 1(1): 11-22.

24. Bland JM, Altman DG. Statistical methods for assessing agreement between two methods of clinical measurement. Lancet 1986; 8: 307-10.

25. Lin LI. A concordance correlation coefficient to evaluate reproducibility. Biometrics 1989; 45(1): 255-68.

26. U.S. Department of Health and Human Services. Centers for Disease Control and Prevention. National Center for Health Statistics. Skinfold Measures, in National Health and Nutrition Examination Survey (NHANES). Hyattsville, MD; December 21, 2006. Disponível em: http://www.cdc.gov/nchs/about/major/nhanes/ Anthropometric\%20Measures.htm. Acessado em 9 Janeiro de 2007.

27. Frisancho AR. New standards of weight and body composition by frame size and height for assessment of nutritional status of adults and the elderly. Am J Clin Nutr 1984; 40(4): 808-19.

28. Ulijaszek SJ, Kerr DA. Anthropometric measurement error and the assessment of nutritional status. Br J Nutr 1999; 82(3): 165-77.

29. Beghetto MG, Fink J, Luft VC, de Mello ED. Estimates of body height in adult inpatients. Clin Nutr 2006; 25(3): 438-43.

30. Klipstein-Grobusch K, Georg T, Boeing H. Interviewer variability in anthropometric measurements and estimates of body composition. Int J Epidemiol 1997; 26(S1): 174-80.

31. Norgan NG, Cameron N. The accuracy of body weight and height recall in middle-aged men. Int J Obes Relat Metab Disord 2000; 24(12): 1695-8.

32. Wada K, Tamakoshi K, Tsunekawa T, Otsuka R, Zhang H, Murata C et al. Validity of self-reported height and weight in a Japanese workplace population. Int J Obes (Lond) 2005; 29(9): 1093-9.

33. Duerksen DR. Teaching medical students the subjective global assessment. Nutrition 2002; 18(4):313-5.

34. Burden ST, Stoppard E, Shaffer J, Makin A, Todd C. Can we use mid upper arm anthropometry to detect malnutrition in medical inpatients? A validation study. $J$ Hum Nutr Diet 2005; 18(4): 287-94 
35. Vegelin AL, Brukx LJ, Waelkens JJ, Van den Broeck J. Influence of knowledge, training and experience of observers on the reliability of anthropometric measurements in children. Ann Hum Biol 2003; 30(1): 6579.

36. Jelliffe DB. The assessment of the nutritional status of the community (with special reference to field surveys in developing regions of the world). Monogr Ser World Health Organ 1966; 53: 3-271.
37. Ferrario M, Carpenter MA, Chambless LE. Reliability of body fat distribution measurements. The ARIC Study baseline cohort results. Atherosclerosis Risk in Communities Study. Int J Obes Relat Metab Disord 1995; 19(7): 449-57.

38. Mueller WH, Taylor WC, Chan W, Sangi-Haghpeykar H, Snider SA, Hsu H. Precision of measuring body fat distribution in adolescent African American girls from the 'Healthy Growth Study'. Am J Hum Biol 1996; 8: 325-9.

Recebido em: 26/03/07

Versão final reapresentada em: 17/09/07

Aprovado em: 29/10/07 